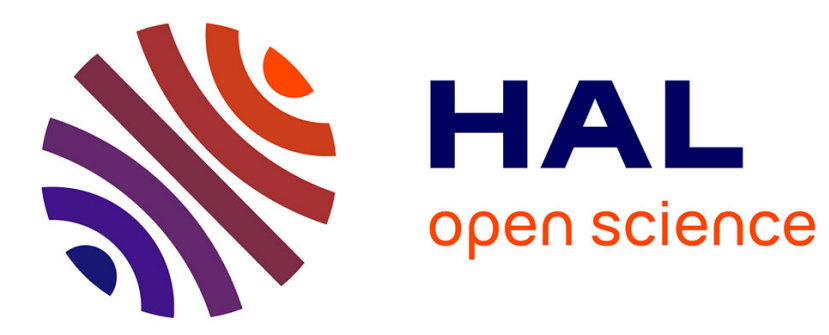

\title{
Design of robust Hinf observers for nonlinear systems using a multiple model
}

Rodolfo Orjuela, Benoît Marx, José Ragot, Didier Maquin

\section{To cite this version:}

Rodolfo Orjuela, Benoît Marx, José Ragot, Didier Maquin. Design of robust Hinf observers for nonlinear systems using a multiple model. 17th IFAC World Congress, Jul 2008, Seoul, South Korea. pp.2756-2761, 10.3182/20080706-5-KR-1001.00464 . hal-00259933

\section{HAL Id: hal-00259933 https://hal.science/hal-00259933}

Submitted on 18 Apr 2014

HAL is a multi-disciplinary open access archive for the deposit and dissemination of scientific research documents, whether they are published or not. The documents may come from teaching and research institutions in France or abroad, or from public or private research centers.
L'archive ouverte pluridisciplinaire HAL, est destinée au dépôt et à la diffusion de documents scientifiques de niveau recherche, publiés ou non, émanant des établissements d'enseignement et de recherche français ou étrangers, des laboratoires publics ou privés. 


\title{
Design of robust $\mathcal{H}_{\infty}$ observers for nonlinear systems using a multiple model
}

\author{
Rodolfo Orjuela, Benoît Marx, José Ragot and Didier Maquin \\ Centre de Recherche en Automatique de Nancy \\ UMR 7039, Nancy-Université, CNRS \\ 2, Avenue de la Forêt de Haye, F 54516 Vandœuvre-lès-Nancy, France \\ $\{$ Rodolfo.Orjuela, Benoit.Marx, Jose.Ragot, \\ Didier.Maquin\}@ensem.inpl-nancy.fr
}

\begin{abstract}
A particular class of multiple model, known as decoupled multiple model, is used in order to cope with the state estimation problem of nonlinear systems. This attractive kind of multiple model is characterized by submodels of which the state belong to spaces of various dimensions, in contrast to the popular Takagi-Sugeno multiple model where the dimensions of the state space of the submodels are identical. Thus the decoupled multiple model is suitable for modelling complex systems with variable structure in the operating range and this fact offers promising prospects in the modelling, control and diagnosis of complex non linear systems. An original procedure for designing a proportional observer and a proportionalintegral observer ensuring $\mathcal{H}_{\infty}$ performances is proposed. Sufficient conditions for ensuring the estimation error convergence are derived employing the LMI framework. Comparison between the state estimation provided by both observers is given via a simulation example.
\end{abstract}

Keywords: multiple model, nonlinear systems, decoupled multiple model, state estimation, $\mathrm{P}$ Observer, PI Observer.

\section{INTRODUCTION}

The state estimation of nonlinear systems has received much attention since many years. However, despite the growing efforts made in this domain, this problem remains nowadays unsolved in a general way.

The idea in order to cope with the state estimation of nonlinear systems basically consists in extending the design of the popular Luenberger (proportional) observer, used in the linear system framework, to the nonlinear systems. However, the direct transition from linear systems to nonlinear ones remains delicate. Hence, new modelling techniques have been proposed in order to make "easier" and "elegant" this transition. One among them is the multiple model approach.

A multiple model can be viewed as a set of submodels, often linear models, combined between them thanks to an interpolation mechanism. In this modelling strategy, each submodel captures the dynamic behaviour of the nonlinear system in a particular operating zone and the interpolation mechanism, employed for taking into consideration the contribution of each submodel, is a set of weighting functions that range between zero and one.

A multiple model is then able to provide an accurate approximation of complex systems by increasing the number of submodels. On the other hand, most of the existing tools in linear systems framework can be partially extended to nonlinear systems represented by a multiple model, of course the submodels must be linear models. Let us notice that the multiple model approach can easily be related to the operating regime based modelling framework [MurraySmith and Johansen, 1997] and to the fuzzy modelling framework [Takagi and Sugeno, 1985].

In the multiple model framework, two major structures can be distinguished in order to build a multiple model [Filev, 1991]. In the first structure, the submodels share the same state vector (Takagi-Sugeno multiple model); in the second one, the submodels are decoupled and their state vectors are different (decoupled multiple model). Consequently, this last multiple model offers flexibility in the modelling step because the submodel dimensions can be adapted to the complexity of each operating zone.

The Takagi-Sugeno model has been successfully used for modelling the dynamic behaviour of nonlinear systems. The observer design based on this multiple model has been largely addressed [Tanaka and Sugeno, 1992, Guerra et al., 2006, Ting, 2006]. The classically used state estimator is an extension of the proportional observer. However, some other classes of state estimators have been proposed, for instance, sliding mode observers [Bergstern and Driankov, 2002] and unknown inputs observer for Takagi-Sugeno descriptor systems [Marx et al., 2007].

By comparison with the Takagi-Sugeno multiple model, the decoupled multiple model has been poorly considered in the literature. However, a few works in the control domain [Gawthrop, 1995, Gatzke and Doyle III, 1999, Gregorcic and Lightbody, 2000] and in modelling [Venkat et al., 2003, Thiaw et al., 2007] of nonlinear systems have made a successful implementation of this structure and shown its relevance. The design of a proportional 
observer based on this multiple model has been recently investigated in [Orjuela et al., 2008].

This paper deals with the design of state estimators of nonlinear system modelled by a decoupled multiple model. Our main contributions are the extension of a previous work in order to design a Proportional Observer (PO) by ensuring $\mathcal{H}_{\infty}$ performance on the one hand and; the design of a Proportional-Integral Observer (PIO), based on this multiple model, that seems not reported previously on the other hand.

The outline of this paper is as follows. The two classic structures of a multiple model are detailed and compared in section 2. Preliminaries and notations are presented in section 3 . In section 4 , the state estimation problem is investigated using a $\mathrm{PO}$ and a PIO. The gains of the observers are obtained by LMI optimization. Finally, in section 5, a simulation example illustrates the state estimation of a decoupled multiple model.

\section{STRUCTURES OF MULTIPLE MODELS}

A multiple model is built by judiciously taking into account the contribution of different submodels. Two basic structures of multiple models can be distinguished according to the use of a single state vector or not by the submodels.

Concerning the identification step, there exists different techniques for the parameter estimation of the submodels considering a particular multiple model structure. See [Murray-Smith and Johansen, 1997, Gasso et al., 2001, Venkat et al., 2003, Orjuela et al., 2006] and the references therein for further information about these techniques.

\subsection{Takagi-Sugeno multiple model}

The structure of the Takagi-Sugeno multiple model is given by [Murray-Smith and Johansen, 1997]:

$$
\begin{aligned}
& \dot{x}(t)=\sum_{i=1}^{L} \mu_{i}(\xi(t))\left\{A_{i} x(t)+B_{i} u(t)\right\}, \\
& y(t)=\sum_{i=1}^{L} \mu_{i}(\xi(t))\left\{C_{i} x(t)\right\}+W \omega(t),
\end{aligned}
$$

where $x \in \mathbb{R}^{n}$ is the state vector, $u \in \mathbb{R}^{m}$ the control input, $y \in \mathbb{R}^{p}$ the output, $\omega \in \mathbb{R}^{q}$ the measurement noise and $A_{i} \in \mathbb{R}^{n \times n}, B_{i} \in \mathbb{R}^{n \times m}, C_{i} \in \mathbb{R}^{p \times n}$ and $W \in \mathbb{R}^{p \times q}$ are known and constant matrices of appropriate dimensions.

The so called decision variable $\xi(t)$ is a perfectly known and accessible signal, for example, the control input and/or a measurable output of the system.

The $\mu_{i}(\xi(t))$ are the weighting functions that ensure the transition between the submodels. They satisfy the following convex sum properties:

$$
\begin{aligned}
& \sum_{i=1}^{L} \mu_{i}(\xi(t))=1, \quad \forall t \\
& 0 \leq \mu_{i}(\xi(t)) \leq 1 . \quad \forall i=1 \ldots L, \quad \forall t
\end{aligned}
$$

The reader may have noticed that the Takagi-Sugeno multiple model can be regarded as a variable parameter model. Indeed, in this multiple model, the contribution of each submodel is taken into consideration thanks to a blend between the parameters of the submodels. Therefore, a common state space is shared by all submodels.

\subsection{Decoupled multiple model}

The structure of the decoupled multiple model is given by [Filev, 1991]:

$$
\begin{aligned}
\dot{x}_{i}(t) & =A_{i} x_{i}(t)+B_{i} u(t), \\
y_{i}(t) & =C_{i} x_{i}(t), \\
y(t) & =\sum_{i=1}^{L}\left\{\mu_{i}(\xi(t)) y_{i}(t)\right\}+W \omega(t),
\end{aligned}
$$

where $x_{i} \in \mathbb{R}^{n_{i}}$ and $y_{i} \in \mathbb{R}^{p}$ are respectively the state vector and the output of the $i^{\text {th }}$ submodel; $y \in \mathbb{R}^{p}$ is the output of the multiple model. The known and constant matrices $A_{i} \in \mathbb{R}^{n_{i} \times n_{i}}, B_{i} \in \mathbb{R}^{n_{i} \times m}, C_{i} \in \mathbb{R}^{p \times n_{i}}$ and $W \in \mathbb{R}^{p \times q}$ are of appropriate dimensions.

Let us notice that in this multiple model no blend between the parameters of the submodels is performed. Indeed, the submodel contribution is taken into account via a weighted sum between the submodel outputs and consequently the submodels do not share the same state space. Hence, the main feature of this multiple model is that the dimension (i.e. the number of states) of the submodels can be different and thanks to this fact, complex systems with variable structure in the operating range can be well modelled.

Note that the outputs $y_{i}(t)$ of the submodels are artificial modelling signals only used in order to provide an approximation of the output of the real system. Therefore the outputs $y_{i}(t)$ cannot be employed as accessible signals for driving an observer.

\section{PRELIMINARIES AND NOTATIONS}

For the simplicity of mathematics manipulations, let us introduce the following augmented state vector:

$$
x(t)=\left[x_{1}^{T}(t) \cdots x_{i}^{T}(t) \cdots x_{L}^{T}(t)\right]^{T} \in \mathbb{R}^{n}, n=\sum_{i=1}^{L} n_{i} .
$$

Now, the decoupled multiple model (3) may be rewritten in the following compact form:

$$
\begin{aligned}
& \dot{x}(t)=\tilde{A} x(t)+\tilde{B} u(t), \\
& y(t)=\tilde{C}(t) x(t)+W \omega(t),
\end{aligned}
$$

where:

$$
\begin{gathered}
\tilde{A}=\left[\begin{array}{ccccc}
A_{1} & 0 & 0 & 0 & 0 \\
0 & \ddots & 0 & 0 & 0 \\
0 & 0 & A_{i} & 0 & 0 \\
0 & 0 & 0 & \ddots & 0 \\
0 & 0 & 0 & 0 & A_{L}
\end{array}\right], \tilde{B}=\left[\begin{array}{c}
B_{1} \\
\vdots \\
B_{i} \\
\vdots \\
B_{L}
\end{array}\right], \\
\tilde{C}(t)=\left[\mu_{1}(\xi(t)) C_{1} \cdots \mu_{i}(\xi(t)) C_{i} \cdots \mu_{L}(\xi(t)) C_{L}\right] .
\end{gathered}
$$

Let us notice that the matrix $\tilde{C}(t)$ can be rewritten as follows: 


$$
\tilde{C}(t)=\sum_{i=1}^{L} \mu_{i}(\xi(t)) \tilde{C}_{i},
$$

where $\tilde{C}_{i}$ is a constant bloc matrix given by:

$$
\tilde{C}_{i}=\left[\begin{array}{lllll}
0 & \cdots & C_{i} & \cdots & 0
\end{array}\right] .
$$

Besides, for convenience, the following notations will be used all along this paper. $P>0(P<0)$ denotes a positive (negative) definite matrix $P ; X^{T}$ denotes the transpose of matrix $X$ and $\mathrm{I}$ is the identity matrix of appropriate dimension. The $L_{2}$-norm of a signal, quantifying its energy is denoted and defined by $\|e(t)\|_{2}^{2}=\int_{0}^{\infty} e^{T}(t) e(t) d t$. We shall simply write $\mu_{i}(\xi(t))=\mu_{i}(t)$.

\section{STATE ESTIMATION}

This section deals with the $\mathcal{H}_{\infty}$ estimation problem, based on a decoupled multiple model, using a PO on the one hand, and a PIO on the other hand. Conditions for ensuring stability and optimal noise attenuation of both observers are established in LMIs terms [Boyd et al., 1994] using the Lyaounov method.

\subsection{Design of a proportional observer}

The design of a PO based on the decoupled multiple model has been investigated in a previous work [Orjuela et al., 2008]. These results are extended in this note in order to ensure $\mathcal{H}_{\infty}$ performances of the estimation.

The proposed PO is given by:

$$
\begin{aligned}
& \dot{\hat{x}}(t)=\tilde{A} \hat{x}(t)+\tilde{B} u(t)+\tilde{K}(y(t)-\hat{y}(t)), \\
& \hat{y}(t)=\tilde{C}(t) \hat{x}(t),
\end{aligned}
$$

where $\hat{x}(t)$ is the state estimation and $\hat{y}(t)$ the output estimation and $\tilde{K} \in \mathbb{R}^{n \times p}$ is the observer gain to be determined.

Define the state estimation error by:

$$
e(t)=x(t)-\hat{x}(t)
$$

and its time-derivative by:

$$
\dot{e}(t)=A_{\text {obs }}(t) e(t)-\tilde{K} W \omega(t),
$$

where $A_{o b s}(t)$ is given by:

$$
\begin{aligned}
A_{o b s}(t) & =\sum_{i=1}^{L} \mu_{i}(t) \phi_{i}, \\
\phi_{i} & =\tilde{A}-\tilde{K} \tilde{C}_{i} .
\end{aligned}
$$

Remark 1. It is easy to notice, from equation (10), that the measurement noise affects directly the estimation error. Indeed, the impact of the measurement noise on the estimation error is directly modified by the observer gain $\tilde{K}$.

The design of the PO (8) consists in finding a matrix $\tilde{K}$ such as the estimation error (9) satisfies the following $\mathcal{H}_{\infty}$ performances:

$$
\begin{gathered}
\lim _{t \rightarrow \infty} e(t)=0 \text { for } \omega(t)=0, \\
\|e(t)\|_{2}^{2} \leq \gamma^{2}\|\omega(t)\|_{2}^{2} \text { for } \omega(t) \neq 0 \text { and } e(0)=0,
\end{gathered}
$$

where $\gamma$ is the $L_{2}$ gain from $\omega(t)$ to $e(t)$ to be minimised. Remark 2. It is well known, in the multiple model framework, that the stability of submodels does not guarantee the stability of the submodel combination. For example, the individual stability of matrices $\phi_{i}$, given by (12), is not sufficient in order to guarantee the stability of the time-varying matrix $A_{o b s}(t)$ given by (11). Consequently, the classic PO design cannot be employed directly in order to obtain the gain matrix $\tilde{K}$. Indeed, the blending between the submodels must be taken into account in the observer design in order to ensure the convergence of the estimation error (9). Classically, the Lyapunov method is used in order to cope with this problem.

Assumption 1. The measurement noise is a bounded energy signal, i.e. $\|\omega(t)\|_{2}^{2}<\infty$.

Theorem 1. The optimal PO (8) for the decoupled multiple model (5), under $\mathcal{H}_{\infty}$ constraints (13), is obtained if there exists a symmetric, positive definite matrix $P$ and a matrix $G$ minimizing $\bar{\gamma}>0$ under the following LMIs

$$
\left[\begin{array}{cc}
\mathcal{A}_{i}+\mathcal{A}_{i}^{T}+\mathrm{I} & \mathcal{B} \\
\mathcal{B}^{T} & -\bar{\gamma} \mathrm{I}
\end{array}\right]<0, \quad i=1 \ldots L
$$

where

$$
\begin{aligned}
\mathcal{A}_{i} & =P \tilde{A}-G \tilde{C}_{i}, \\
\mathcal{B} & =-G W .
\end{aligned}
$$

The observer gain is given by $\tilde{K}=P^{-1} G$ and the $L_{2}$ gain from $\omega(t)$ to $e(t)$ is given by $\gamma=\sqrt{\bar{\gamma}}$.

Proof. Consider the quadratic Lyapunov function:

$$
V(t)=e^{T}(t) P e(t), \quad P>0 \quad P=P^{T},
$$

and $\gamma>0$ such that

$$
\dot{V}(t)<-e^{T}(t) e(t)+\gamma^{2} \omega^{T}(t) \omega(t), \quad \forall t .
$$

Notice that the integration of both sides of (16) yields:

$$
\int_{0}^{\infty} \dot{V}(t) d t<-\int_{0}^{\infty} e^{T}(t) e(t) d t+\gamma^{2} \int_{0}^{\infty} \omega^{T}(t) \omega(t) d t
$$

that it is also equivalent to:

$$
V(\infty)-V(0)<-\|e(t)\|_{2}^{2}+\gamma^{2}\|w(t)\|_{2}^{2},
$$

and by taking into account the fact that $V(\infty)>0$ and $V(0)=0$, the above inequality becomes:

$$
\|e(t)\|_{2}^{2}<\gamma^{2}\|w(t)\|_{2}^{2},
$$

hence the attenuation level between the measurement noise and the estimation error, given by (13b), is guaranteed if condition (16) is fulfilled.

Now, conditions verifying (16) must be established in order to satisfy conditions (13). The time-derivative of (15) along the trajectories of (10) is given by:

$$
\dot{V}(t)=\dot{e}^{T}(t) P e(t)+e^{T}(t) P \dot{e}(t),
$$

that becomes using (10): 


$$
\begin{aligned}
\dot{V}(t) & =e^{T}(t)\left(A_{o b s}^{T}(t) P+P A_{o b s}(t)\right) e(t) \\
& -\omega^{T}(t)(\tilde{K} W)^{T} P e(t)-e^{T}(t) P \tilde{K} W \omega(t) .
\end{aligned}
$$

The above equation can be rewritten as:

$$
\dot{V}(t)=\psi(t)^{T} \Omega(t) \psi(t)
$$

where

$$
\begin{aligned}
& \Omega(t)=\left[\begin{array}{cc}
A_{o b s}^{T}(t) P+P A_{o b s}(t) & -P \tilde{K} W \\
-(\tilde{K} W)^{T} P & 0
\end{array}\right], \\
& \psi(t)=\left[e^{T}(t) \omega^{T}(t)\right]^{T} .
\end{aligned}
$$

Now, in order to ensure that (16) for shall satisfy the following condition:

$$
\psi^{T}(t)\left\{\Omega(t)+\left[\begin{array}{cc}
\mathrm{I} & 0 \\
0 & -\gamma^{2} \mathrm{I}
\end{array}\right]\right\} \psi(t)<0,
$$

which is a quadratic form in $\psi(t)$. Hence, by using (23) and (11), the negativity of (25) is satisfied if:

$$
\sum_{i=1}^{L} \mu_{i}(t)\left[\begin{array}{cc}
\left(\tilde{A}-\tilde{K} \tilde{C}_{i}\right)^{T} P+P\left(\tilde{A}-\tilde{K} \tilde{C}_{i}\right)+\mathrm{I} & -P \tilde{K} W \\
-(\tilde{K} W)^{T} P & -\gamma^{2} \mathrm{I}
\end{array}\right]<0
$$

Now, according to the convex sum properties (2b), the above inequality is also satisfied if:

$$
\left[\begin{array}{cc}
\left(\tilde{A}-\tilde{K} \tilde{C}_{i}\right)^{T} P+P\left(\tilde{A}-\tilde{K} \tilde{C}_{i}\right)+\mathrm{I} & -P \tilde{K} W \\
-(\tilde{K} W)^{T} P & -\gamma^{2} \mathrm{I}
\end{array}\right]<0
$$

for $i=1 \ldots L$.

Finally, let us notice that (27) is not a LMI in $P, \tilde{K}$ and $\gamma$. However, it becomes a strict LMI by setting $G=P \tilde{K}$ and $\bar{\gamma}=\gamma^{2}$. Now, standard convex optimization algorithms can be used to find matrices $P$ and $G$ for a minimal value of $\bar{\gamma}$.

Note also that the condition (14) in the theorem 1 implies:

$$
\left(P \tilde{A}-G \tilde{C}_{i}\right)+\left(P \tilde{A}-G \tilde{C}_{i}\right)^{T}<0, \quad i=1 \ldots L,
$$

which means that the matrix $A_{o b s}(t)$, given by (11), is Hurwitz for any blend between the submodel outputs [Orjuela et al., 2008]. Hence, the asymptotic convergence of the estimation error is ensured under $\omega(t)=0$ and condition (13a) is therefore satisfied.

\subsection{Design of a proportional-integral observer}

The concept of PIO proposed in [Beale and Shafai, 1989] can be extended in order to provide a robust state estimation of a nonlinear system. In a PIO, an integral term of the estimation error is taken into account via a supplementary variable $z(t)$. Hence, thanks to this extra integral variable a robustness degree of the state estimation with respect to the plant perturbation is achieved [Weinmann, 1991]. Our approach for designing the PIO is similar to the approach proposed in [Hua and Guan, 2005] used in the synchronization of a chaotic system.

Hence, the decoupled multiple model (5) becomes:

$$
\begin{aligned}
& \dot{x}(t)=\tilde{A} x(t)+\tilde{B} u(t), \\
& \dot{z}(t)=\tilde{C}(t) x(t)+W \omega(t), \\
& y(t)=\tilde{C}(t) x(t)+W \omega(t),
\end{aligned}
$$

where $z(t)=\int_{0}^{t} y(\xi) d \xi$.

The above equations can be rewritten in the following augmented form:

$$
\begin{aligned}
\dot{x}_{a}(t) & =\tilde{A}_{1}(t) x_{a}(t)+\bar{C}_{1} \tilde{B} u(t)+\bar{C}_{2} W \omega(t), \\
y(t) & =\tilde{C}(t) \bar{C}_{1}^{T} x_{a}(t)+W \omega(t) \\
z(t) & =\bar{C}_{2}^{T} x_{a}(t)
\end{aligned}
$$

where

$$
x_{a}(t)=\left[\begin{array}{l}
x(t) \\
z(t)
\end{array}\right], \tilde{A}_{1}(t)=\left[\begin{array}{cc}
\tilde{A} & 0 \\
\tilde{C}(t) & 0
\end{array}\right], \bar{C}_{1}=\left[\begin{array}{l}
\mathrm{I} \\
0
\end{array}\right], \bar{C}_{2}=\left[\begin{array}{l}
0 \\
\mathrm{I}
\end{array}\right] .
$$

The state estimation of the decoupled multiple model (30) is achieved via the following PIO:

$$
\begin{aligned}
\dot{\hat{x}}_{a}(t)= & \tilde{A}_{1}(t) \hat{x}_{a}(t)+\bar{C}_{1} \tilde{B} u(t)+K_{P}(y(t)-\hat{y}(t)) \\
& \quad+K_{I}(z(t)-\hat{z}(t)) \\
\hat{y}(t) & =\tilde{C}(t) \bar{C}_{1}^{T} \hat{x}_{a}(t), \\
\hat{z}(t) & =\bar{C}_{2}^{T} \hat{x}_{a}(t) .
\end{aligned}
$$

Notice that the use of the integral action $z(t)$ is at the origin of the designation Proportional-Integral Observer.

Define the state estimation error by:

$$
e_{a}(t)=x_{a}(t)-\hat{x}_{a}(t),
$$

and its time-derivative by:

$$
\dot{e}_{a}(t)=\tilde{A}_{o b s}(t) e_{a}(t)+\left(\bar{C}_{2} W-K_{P} W\right) \omega(t),
$$

where $\tilde{A}_{o b s}(t)$ is defined by:

$$
\tilde{A}_{\text {obs }}(t)=\tilde{A}_{1}(t)-K_{P} \tilde{C}(t) \bar{C}_{1}^{T}-K_{I} \bar{C}_{2}^{T} .
$$

Remark 3. It is easy to see, from equation (34), that the attenuation of the measurement noise can be adjusted via the choice of $K_{P}$.

Let us notice that, by taking into account the form (6) of $\tilde{C}(t)$, the matrix $\tilde{A}_{1}(t)$ becomes:

$$
\tilde{A}_{1}(t)=\sum_{i=1}^{L} \mu_{i}(t) \bar{A}_{i}
$$

where

$$
\bar{A}_{i}=\left[\begin{array}{cc}
\tilde{A} & 0 \\
\tilde{C}_{i} & 0
\end{array}\right]
$$

Finally, by using (6) and (36), the matrix $\tilde{A}_{o b s}(t)$ can be rewritten as:

$$
\begin{aligned}
\tilde{A}_{o b s}(t) & =\sum_{i=1}^{L} \mu_{i}(t) \Phi_{i}, \\
\Phi_{i} & =\bar{A}_{i}-K_{P} \tilde{C}_{i} \bar{C}_{1}^{T}-K_{I} \bar{C}_{2}^{T} .
\end{aligned}
$$


Theorem 2. The optimal PIO (32) for the decoupled multiple model (30), under $\mathcal{H}_{\infty}$ constraints (13), is obtained if there exist a symmetric, positive definite matrix $P$ and matrices $L_{P}$ and $L_{I}$ minimizing $\bar{\gamma}>0$ under the following LMIs

$$
\left[\begin{array}{cc}
\mathcal{A}_{i}+\mathcal{A}_{i}^{T}+\mathrm{I} & \mathcal{B} \\
\mathcal{B}^{T} & -\bar{\gamma} \mathrm{I}
\end{array}\right]<0, \quad i=1 \ldots L
$$

where

$$
\begin{aligned}
\mathcal{A}_{i} & =P \bar{A}_{i}-L_{P} \tilde{C}_{i} \bar{C}_{1}^{T}-L_{I} \bar{C}_{2}^{T}, \\
\mathcal{B} & =P \bar{C}_{2} W-L_{P} W .
\end{aligned}
$$

The observer gains are given by $K_{P}=P^{-1} L_{P}$ and $K_{I}=P^{-1} L_{I}$; the $L_{2}$ gain from $\omega(t)$ to $e(t)$ is given by $\gamma=\sqrt{\bar{\gamma}}$.

Proof. The proof of the above theorem is omitted here. Indeed, this proof is carried out in a similar way to the previous case.

\section{SIMULATION EXAMPLE}

Considerer the decoupled multiple model with $L=2$ different dimension submodels given by:

$$
\begin{array}{rlrl}
A_{1} & =\left[\begin{array}{ccc}
-2.0 & 0.5 & 0.6 \\
-0.3 & -0.9 & -0.5 \\
-1.0 & 0.6 & -0.8
\end{array}\right], & & A_{2}=\left[\begin{array}{cc}
-0.8 & -0.4 \\
0.1 & -1.0
\end{array}\right], \\
B_{1} & =\left[\begin{array}{lll}
1.0 & 0.8 & 0.5
\end{array}\right]^{T}, & B_{2} & =\left[\begin{array}{cc}
-0.5 & 0.8
\end{array}\right], \\
C_{1} & =\left[\begin{array}{ccc}
0.9 & -0.8 & -0.5 \\
-0.4 & 0.6 & 0.7
\end{array}\right], & C_{2}=\left[\begin{array}{cc}
-0.8 & 0.6 \\
0.4 & -0.7
\end{array}\right], \\
W & =\left[\begin{array}{cc}
0.4 & 0 \\
0 & -0.3
\end{array}\right] . & &
\end{array}
$$

Here, the decision variable $\xi(t)$ is the input signal $u(t) \in[0,1]$. The weighting functions are obtained from normalised Gaussian functions:

$$
\begin{aligned}
& \mu_{i}(\xi(t))=\eta_{i}(\xi(t)) / \sum_{j=1}^{L} \eta_{j}(\xi(t)), \\
& \eta_{i}(\xi(t))=\exp \left(-\left(\xi(t)-c_{i}\right)^{2} / \sigma^{2}\right),
\end{aligned}
$$

with the standard deviation $\sigma=0.5$ and the centres $c_{1}=0.25$ and $c_{2}=0.75$. The measurement noise $\omega(t)$ is a normally distributed random signal with zero mean and standard deviation equal to one. The input, the weighting functions and the outputs are shown in figure 1 .

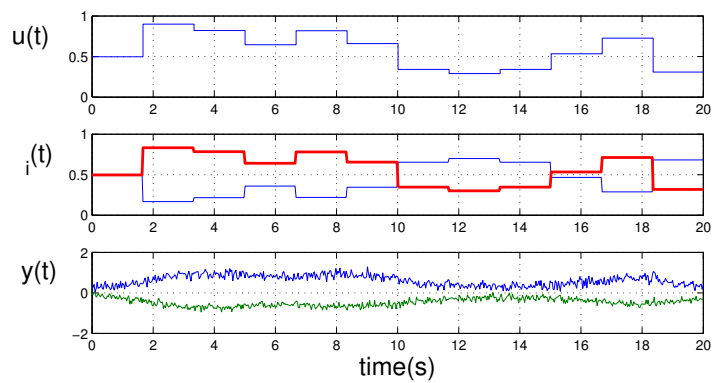

Fig. 1. Input, weighting functions and outputs

A solution to conditions of theorems 1 and 2 can be found by using, for example, YALMIP interface [Löfberg, 2004] coupled to SeDuMi solver. Conditions of theorem 1 are fulfilled with:

$$
\tilde{K}=\left[\begin{array}{ccccc}
0.193 & -0.030 & 0.092 & -0.032 & -0.014 \\
0.224 & 0.113 & 0.220 & -0.016 & -0.027
\end{array}\right]^{T}
$$

with an attenuation index $\gamma^{2}=2$. The obtained matrix $P$ is given in appendix (matrix $P_{P}$ ).

Conditions of theorem 2 are fulfilled with:

$$
\begin{aligned}
K_{P} & =\left[\begin{array}{ccccccc}
0.004 & -0.035 & -0.007 & -0.017 & -0.003 & 0.934 & -0.032 \\
0.017 & 0.027 & 0.030 & 0.007 & -0.005 & 0.002 & 0.941
\end{array}\right]^{T}, \\
K_{I} & =\left[\begin{array}{lllllll}
0.142 & 0.568 & 0.131 & 0.050 & 0.008 & 2.626 & 0.017 \\
0.139 & 0.519 & 0.156 & -0.010 & 0.008 & 0.017 & 2.496
\end{array}\right]^{T},
\end{aligned}
$$

with an attenuation index $\gamma^{2}=0.1$. The obtained matrix $P$ is given in appendix (matrix $P_{P I}$ ).

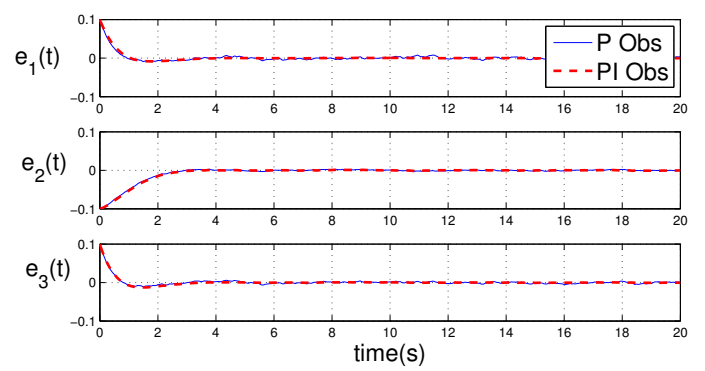

Fig. 2. State estimation errors of submodel 1
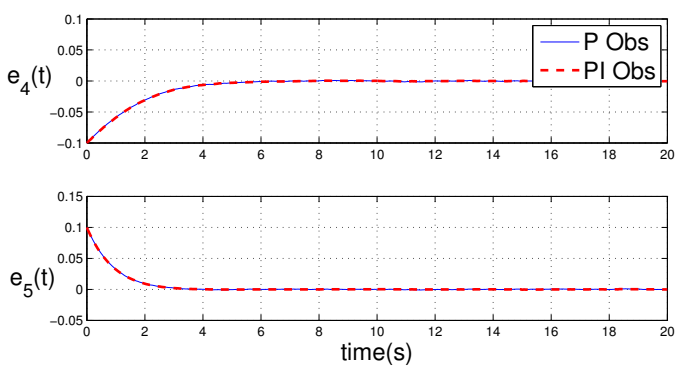

Fig. 3. State estimation errors of submodel 2

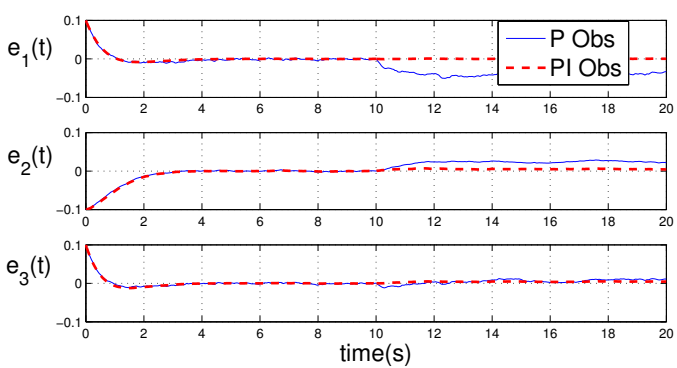

Fig. 4. State estimation errors of submodel 1 under perturbation
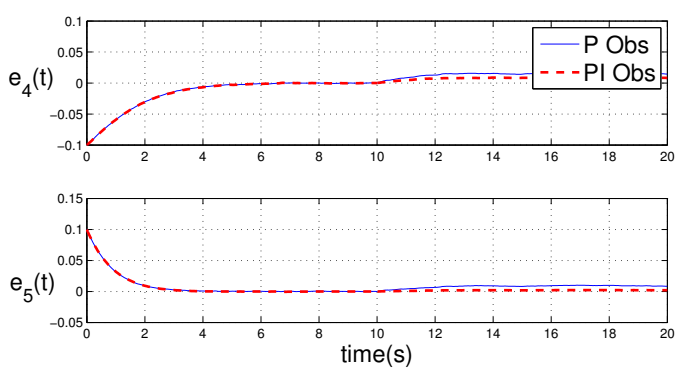

Fig. 5. State estimation errors of submodel 2 under perturbation 
It can be noted the gap between the attenuation levels obtained with the PO and the PIO. The state estimation errors obtained using the proposed PO and PIO are depicted in figures 2 and 3 . Let us notice that the initial conditions of the multiple model are not null and the initial conditions of the observers are null.

Now, a constant perturbation equal to 0.5 is added to the output $y_{1}(t)$ at $t=10$. This perturbation can be due, for example, to a sensor fault. The estimation errors provided by both observers are plotted in figures 4 and 5 . As clearly seen from these pictures, the PIO provides the best state estimation under the considered perturbation.

\section{CONCLUSION}

This paper has proposed a new design of a proportional observer and a proportional-integral observer, based on a decoupled multiple model approach, for estimating the state of nonlinear systems. The decoupled multiple model is suitable for modelling complex systems with variable structure in the operating range. Indeed, in this multiple model the dimension of the submodels can be adapted to the complexity of the operating zones because each submodel has a different state vector. The effectiveness of the proposed approach and a comparison between both observers are illustrated via a simulation example.

\section{APPENDIX}

Matrices $P_{P}$ and $P_{P I}$, solutions to conditions of theorem 1 and 2 are given respectively by:

$$
\begin{aligned}
P_{P}= & {\left[\begin{array}{cccccc}
3.33 & -0.14 & -2.68 & 0.12 & -0.07 \\
-0.14 & 3.89 & -1.00 & -0.25 & 0.51 \\
-2.68 & -1.00 & 5.44 & 0.12 & -0.24 \\
0.12 & -0.25 & 0.12 & 11.50 & -10.19 \\
-0.07 & 0.51 & -0.24 & -10.19 & 25.69
\end{array}\right], } \\
P_{P I}= & {\left[\begin{array}{cccccccc}
5.196 & -0.576 & -3.554 & 0.058 & 0.010 & 0.043 & 0.076 \\
-0.576 & 5.177 & -0.976 & -0.144 & 0.188 & -0.959 & -0.913 \\
-3.554 & -0.976 & 7.457 & 0.070 & -0.188 & 0.187 & 0.110 \\
0.058 & -0.144 & 0.070 & 8.260 & -6.907 & -0.111 & 0.074 \\
0.010 & 0.188 & -0.188 & -6.907 & 19.939 & 0.054 & -0.124 \\
0.043 & -0.959 & 0.187 & -0.111 & 0.054 & 1.276 & 0.175 \\
0.076 & -0.913 & 0.110 & 0.074 & -0.124 & 0.175 & 1.315
\end{array}\right] . }
\end{aligned}
$$

\section{ACKNOWLEDGEMENTS}

This work is partially supported by the Conseil Régional de Lorraine (France).

\section{REFERENCES}

S. Beale and B. Shafai. Robust control system design with a proportional integral observer. International Journal of Control, 50(1):97-111, 1989.

R. Bergstern, P. Palm and D. Driankov. Observers for Takagi-Sugeno fuzzy systems. IEEE Transactions on Systems, Man, and Cybernetics, 32(1):114-121, 2002.

S. Boyd, L. El Ghaoui, E. Feron, and V. Balakrishnan. Linear Matrix Inequalities in System and Control Theory. SIAM studies in applied mathematics. SIAM, Philadelphia, P.A., 1994.

D. Filev. Fuzzy modeling of complex systems. International Journal of Approximate Reasoning, 5(3):281-290, 1991.
K. Gasso, G. Mourot, and J. Ragot. Structure identification in multiple model representation: elimination and merging of local models. In 40th IEEE Conference on Decision and Control, pages 2992-2997, Orlando, USA, 2001.

E. P. Gatzke and F. J. Doyle III. Multiple model approach for CSTR control. In 14th IFAC World Congress, pages 343-348, Beijing, P. R. China, 1999.

P.J. Gawthrop. Continuous-time local state local model networks. In IEEE Conference on Systems, Man \& Cybernetics, pages 852-857, Vancouver, Canada, 1995.

G. Gregorcic and G. Lightbody. Control of highly nonlinear processes using self-tuning control and multiple/local model approaches. In 2000 IEEE International Conference on Intelligent Engineering Systems, INES 2000, pages 167-171, Portoroz, Slovenie, 2000.

T. M. Guerra, A. Kruszewski, L. Vermeiren, and H. Tirmant. Conditions of output stabilization for nonlinear models in the Takagi-Sugeno's form. Fuzzy Sets and Systems, 157(9):1248-1259, 2006.

C. Hua and X. Guan. Synchronization of chaotic systems based on PI observer design. Physics Letters A, 334 (5-6):382-389, 2005.

J. Löfberg. YALMIP : A toolbox for modeling and optimization in MATLAB. In Computer Aided Control Systems Design, CACSD, pages 284-289, Taipei, Taiwan, 2004.

B. Marx, D. Koenig, and J. Ragot. Design of observers for Takagi-Sugeno descriptor systems with unknown inputs and application to fault diagnosis. IET Control Theory and Applications, 1(5):1487-1495, 2007.

R. Murray-Smith and T.A. Johansen. Multiple model approaches to modelling and control. Taylor \& Francis, London, 1997.

R. Orjuela, D. Maquin, and J. Ragot. Nonlinear system identification using uncoupled state multiple-model approach. In Workshop on Advanced Control and Diagnosis, ACD'2006, Nancy, France, 2006.

R. Orjuela, B. Marx, J. Ragot, and D. Maquin. State estimation for nonlinear systems using decoupled multiple model. International Journal of Modelling Identification and Control, 3(5), 2008. (to be published).

M. Takagi and M. Sugeno. Fuzzy identification of systems and its application to modelling and control. IEEE Transactions on Systems Man and Cybernetics, 15(1): 116-132, 1985.

K. Tanaka and M. Sugeno. Stability analysis and desing of fuzzy control systems. Fuzzy Sets and Systems, 45 (2):135-156, 1992.

L. Thiaw, K. Madani, R. Malti, and G. Sow. Implementation of recurrent multi-models for system identification. In 4th International Conference on Informatics in Control, Automation and Robotics, ICINCO, pages 314-321, Angers, France, 2007.

C. Ting. Stability analysis and design of Takagi-Sugeno fuzzy systems. Information Sciences, 176(19):2817$2845,2006$.

A.N. Venkat, P. Vijaysai, and R.D. Gudi. Identification of complex nonlinear processes based on fuzzy decomposition of the steady state space. Journal of Process Control, 13(6):473-488, 2003.

A. Weinmann. Uncertain Models and Robust Control. Springer-Verlag Wien New York, Vienne, 1991. 